\title{
Rolipram, a Selective Inhibitor of Phosphodiesterase Type 4, Pronouncedly Enhanced the Forskolin-Induced Promotion of Dopamine Biosynthesis in Primary Cultured Rat Mesencephalic Neurons
}

\author{
Nobuyuki Yamashita, Mio Miyashiro, Jun Baba and Aiko Sawa* \\ Drug Discovery, Pharmaceutical Research Center, Meiji Seika Kaisha, Ltd., 760 Morooka-cho, Kohoku-ku, Yokohama 222, Japan
}

Received February 13, 1997 Accepted July 15, 1997

\begin{abstract}
A selective inhibitor of cyclic nucleotide phosphodiesterase (PDE) 4, rolipram, markedly enhanced the forskolin-induced increase of intracellular dopamine and dihydroxyphenylacetate (DOPAC, a metabolite of dopamine) levels in primary cultured rat mesencephalic neurons and the forskolin-induced increase of dopamine and DOPAC in extracellular medium. Selective inhibitors of PDE2, PDE3, PDE5 and PDE6 did not have such a promoting effect, and the PDE1 inhibitor vinpocetine and W-7 caused dopamine depletion in the neurons. These findings suggested that PDE4 plays a major role in regulating the intracellular cAMP level to control the dopamine biosynthesis in mesencephalic neurons, whereas PDE1 regulates dopamine release instead.
\end{abstract}

Keywords: Phosphodiesterase, Rolipram, Dopamine biosynthesis

Cyclic adenosine 3',5'-monophosphate (cAMP), a typical second messenger, is produced through the conversion of adenosine triphosphate (ATP) by adenylyl cyclase and is converted to 5'-AMP by cyclic nucleotide phosphodiesterases (PDEs) (1). Seven types of PDE (PDE1-7) have been reported (1). Among them, PDE1, PDE2, PDE3 and PDE4 have been reported to be present in the brain $(1,2)$. PDE4 is a cAMP-specific PDE, whereas the other types of PDE also or specifically hydrolyze cyclic guanosine $3^{\prime}, 5^{\prime}$-monophosphate (cGMP). Thus, inhibition of PDE4 specifically enhances intracellular cAMP accumulation that would lead to potentiation of cellular functions mediated by cAMP in the central nervous system. A PDE4 inhibitor affected the release of noradrenaline and dopamine from rat brain slices (3) and survival of dopaminergic neurons in vitro (4). These facts suggest that PDE4 plays a major role in the regulation of intracellular cAMP in the dopaminergic neuron. The biosynthesis of dopamine is regulated by cAMP through activation of tyrosine hydroxylase (TH), the rate-limiting enzyme in the biosynthesis of dopamine, via activation of cAMP-dependent protein kinase A (PKA) (5). In the present study, we evaluated the effects of rolipram, a selective PDE4 inhibitor, on dopamine biosynthesis in

\footnotetext{
* To whom correspondence should be addressed.
}

comparison with the selective inhibitors for other types of PDE using primary cultured rat mesencephalic neurons with or without the influence of adenylyl cyclase stimulation by forskolin.

The 15-day-old embryos of Wistar rat were obtained from pregnant rats (Japan SLC, Hamamatsu) anesthetized by ether, and mesencephalic cells of the embryos were dissociated by trypsinization and trituration in Dulbecco's modified Eagle's medium containing $1 \%$ glucose (DME; Nissui Pharmaceutical Co., Tokyo) and 10\% fetal bovine serum (FBS; Life Technologies, Inc., Rockville, MD, USA). The cells were seeded onto poly-L-lysine coated, 96-well culture plates (Sumitomo Bakelite Co., Tokyo) at a plating density of $10^{6} \mathrm{cells} / \mathrm{cm}^{2}$ and incubated at $37^{\circ} \mathrm{C}$ in a humidified $5 \% \mathrm{CO}_{2}-95 \%$ air atmosphere. The culture medium in each well was changed twice a week, and the neurons were cultured for 8-9 days before the experiments.

The following selective PDE inhibitors were used at concentrations 3-5 times higher than the reported values of $K_{i}$ or $\operatorname{IC}_{50}(1,6)$ to selectively and sufficiently inhibit each type of PDE: $60 \mu \mathrm{M}$ vinpocetine (a PDE1 inhibitor; Calbiochem-Novabiochem Co., La Jolla, CA, USA); 15 $\mu \mathrm{M}$ erythro-9-(2-hydroxy-3-nonyl) adenine (EHNA, a PDE2 inhibitor; Sigma Chemical Co., St. Louis, MO, USA), $1 \mu \mathrm{M}$ milrinone (a PDE3 inhibitor, Sigma), $1 \mu \mathrm{M}$ 
rolipram (a PDE4 inhibitor; Schering, Berlin, Germany), $10 \mu \mathrm{M}$ Ro 20-1724 (4-[(3-butoxy-4-methoxyphenyl)methyl]-2-imidazolinone, a PDE4 inhibitor; Bio Mol Research Laboratories, Inc., Plymouth Meeting, PA, USA) and $1.5 \mu \mathrm{M}$ zaprinast (PDE5 and PDE6 inhibitor, Sigma).

For intracellular dopamine and dihydroxyphenylacetate (DOPAC, a metabolite of dopamine) measurement, the cells were washed with DME and incubated at $37^{\circ} \mathrm{C}$ with DME containing each PDE inhibitor with or without $0.3 \mu \mathrm{M}$ forskolin, an adenylyl cyclase activator, for 60 min. Then medium was removed and the reaction was terminated by addition of a mixture of $0.1 \mathrm{M} \mathrm{HClO}_{4}$ and $0.03 \mathrm{M}$ EDTA, the cells were sonicated and centrifuged at $7500 \times \mathrm{g}$ for $15 \mathrm{~min}$. Dopamine and DOPAC levels in the supernatants were measured by high performance liquid chromatography (HPLC) with coulometric detection as reported previously (7). Protein contents in the pellets, which were solubilized with $0.1 \mathrm{~N} \mathrm{NaOH}$, was measured with a commercially available protein assay kit (BCA protein assay reagent; Pierce, Rockford, IL, USA).

For extracellular dopamine and DOPAC measurement, the cells were incubated at $37^{\circ} \mathrm{C}$ in balanced salt solution (BSS; $130 \mathrm{mM} \mathrm{NaCl}, 5.4 \mathrm{mM} \mathrm{KCl}, 1.8 \mathrm{mM} \mathrm{CaCl}_{2}, 1.3$ $\mathrm{mM} \mathrm{MgSO} 4,10 \mathrm{mM}$ glucose and $20 \mathrm{mM}$ Hepes, $\mathrm{pH}$ 7.4) containing each PDE inhibitor with or without $0.3 \mu \mathrm{M}$ forskolin for $60 \mathrm{~min}$, and the incubated supernatants were used for measurement of extracellular dopamine and DOPAC with HPLC. In each experiment, the significance of the difference between the untreated group and the control group treated with forskolin alone was determined by Student's or Aspin-Welch's $t$-test. The significance of the difference between the control group and each PDE inhibitor treated group was determined by applying Dunnett's multiple comparison test.

Rolipram $(1 \mu \mathrm{M})$ did not affect the intracellular dopa-

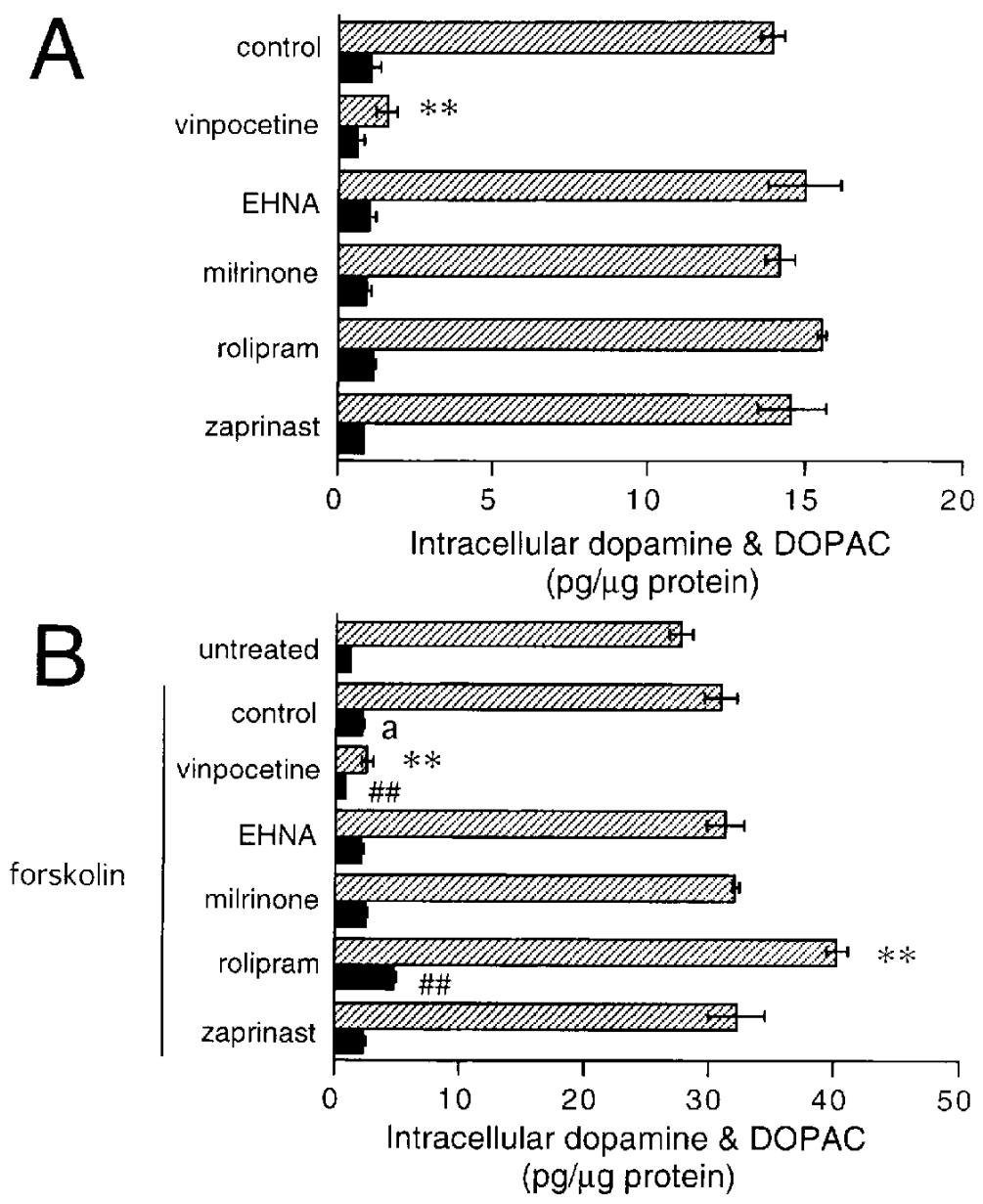

Fig. 1. The effect of inhibitor for each PDE on the levels of dopamine $(\square A)$ and DOPAC $(\square)$ in cultured dopaminergic neurons without (A) or with (B) forskolin. The concentrations of drugs are as follows: $60 \mu \mathrm{M}$ vinpocetine, $15 \mu \mathrm{M}$ EHNA, $1 \mu \mathrm{M}$ milrinone, $1 \mu \mathrm{M}$ rolipram, $1.5 \mu \mathrm{M}$ zaprinast and $0.3 \mu \mathrm{M}$ forskolin. Values each represent a mean \pm S.E.M. $(\mathrm{n}=3$ ) and symbols show the following in each graph: ${ }^{* *} \mathrm{P}<0.01$ and ${ }^{\mu *} \mathrm{P}<0.01$ vs the dopamine and DOPAC level in the control group, respectively, a: $\mathrm{P}<0.01$ vs the DOPAC level in the untreated group. 

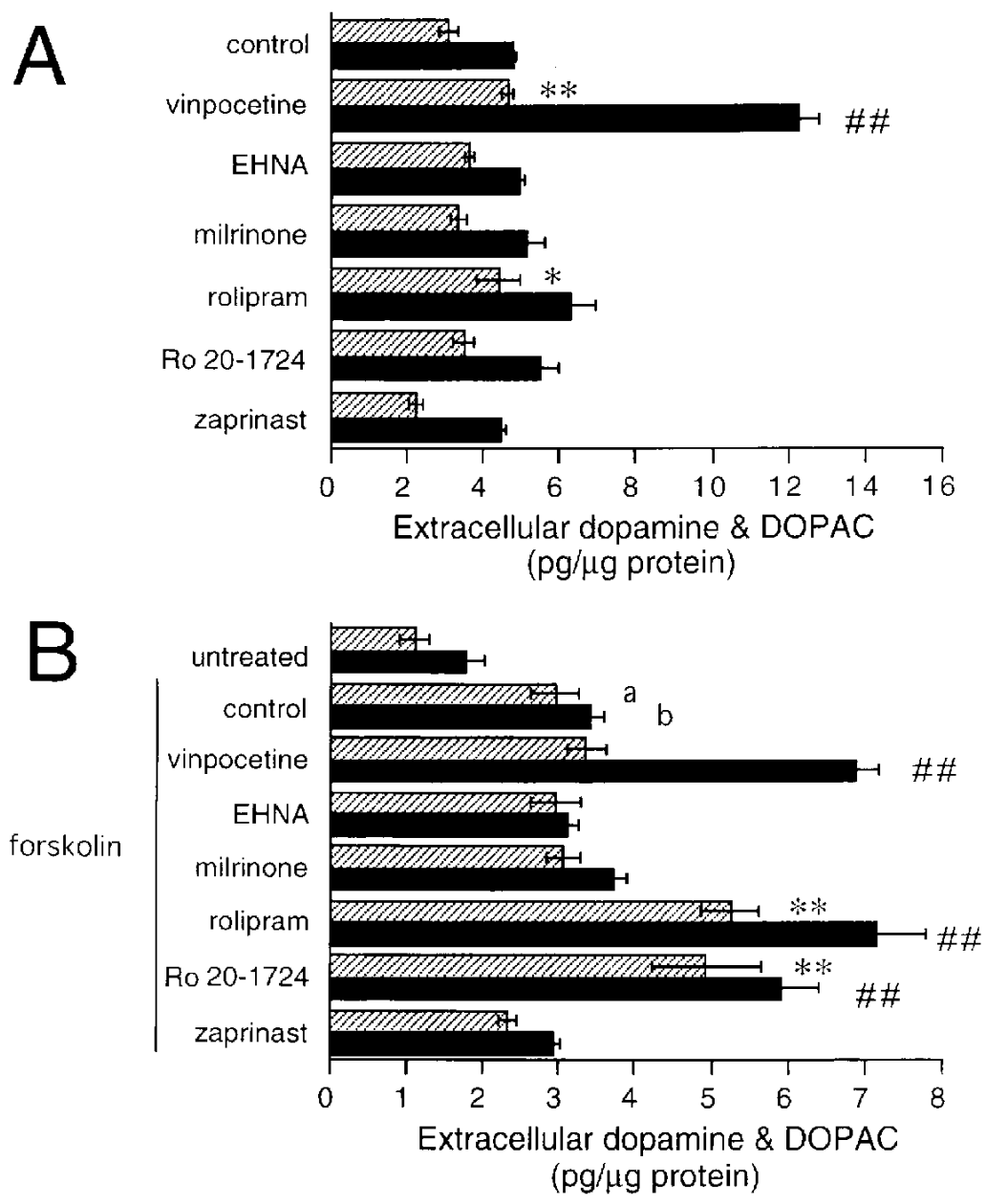

Fig. 2. The effect of inhibitor for each PDE on the amounts of dopamine ( $\triangle$ ) and DOPAC ( $\square$ ) in extracellular medium used for incubation of the dopaminergic neurons without (A) or with (B) forskolin. The concentrations of each PDE inhibitor and forskolin are the same in Fig. 1, except $10 \mu \mathrm{M}$ Ro 20-1724. Values each represent a mean \pm S.E.M. (n=4), and symbols show the following in each graph: ${ }^{*} \mathrm{P}<0.05$ and ${ }^{* *} \mathrm{P}<0.01$ vs the dopamine in the control group, ${ }^{*} \mathrm{P}<0.01$ vs the DOPAC in the control group, "and ${ }^{\mathrm{b}}: \mathrm{P}<0.01$ vs the dopamine and DOPAC levels in the untreated group, respectively.

mine level in the absence of forskolin stimulation (Fig. 1A), but significantly enhanced the forskolin-induced increase in the intracellular dopamine level $(\mathrm{P}<0.01$, Fig. 1B). Similarly, the increase in intracellular DOPAC content was enhanced by rolipram in the presence $(P<0.01$, Fig. 1B), but not in the absence (Fig. 1A), of forskolin. On the other hand, EHNA $(15 \mu \mathrm{M})$, milrinone $(1 \mu \mathrm{M})$ and zaprinast $(1.5 \mu \mathrm{M})$ had no effect on dopamine and DOPAC levels both in the absence or the presence of forskolin stimulation (Fig. 1: A and B). Vinpocetine (60 $\mu \mathrm{M})$ significantly decreased the dopamine level in the absence and presence of forskolin and the DOPAC level in the presence of forskolin ( $\mathrm{P}<0.01$, Fig. 1 : $\mathrm{A}$ and $\mathrm{B})$.

Rolipram and Ro 20-1724 significantly increased the extracellular levels of dopamine and DOPAC in the presence of forskolin $(\mathrm{P}<0.01$, Fig. $2 \mathrm{~B})$. Rolipram also significantly increased the extracellular level of dopa- mine in the absence of forskolin ( $P<0.05$, Fig. $2 A)$. EHNA, milrinone and zaprinast had no effect on dopamine and DOPAC levels in the absence or the presence of forskolin stimulation (Fig. 2: A and B). Vinpocetine significantly increased the extracellular dopamine and DOPAC levels in the absence of forskolin $(P<0.01$, Fig. $2 \mathrm{~A})$ and significantly increased the extracellular DOPAC in the presence of forskolin ( $\mathrm{P}<0.01$, Fig. $2 \mathrm{~B}$ ).

We also investigated the effects of W-7 $(100 \mu \mathrm{M})$, a calmodulin antagonist, and found that it decreased intracellular dopamine ( $4 \%$ of control) and increased extracellular dopamine ( $400 \%$ of control) without any effect on the level of intracellular and extracellular DOPAC (data not shown).

The enhancing effect of rolipram on the forskolininduced increase of intracellular dopamine and DOPAC levels was concentration dependent in the range of 1-100 


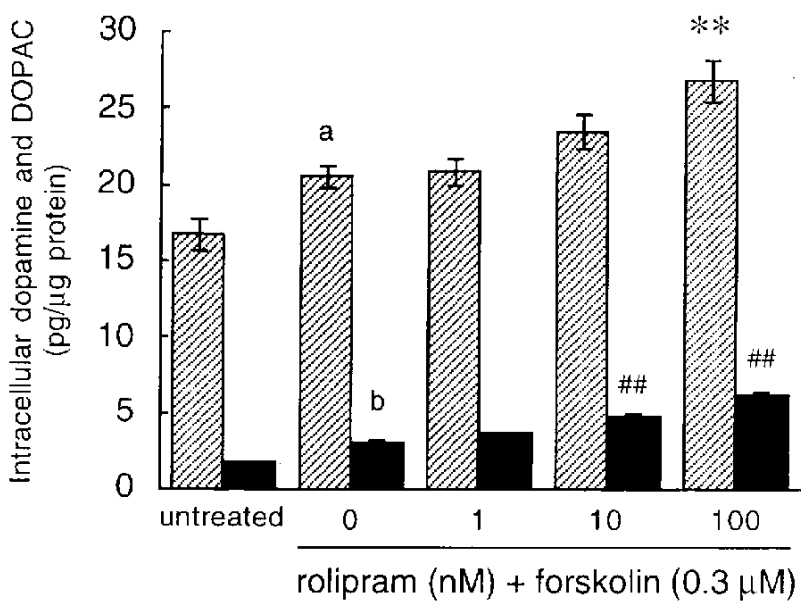

Fig. 3. Effects of rolipram on the dopamine ( $\triangle)$ and DOPAC ( $)$ levels in the cultured dopaminergic neurons stimulated with forskolin. The cells were treated with rolipram at the concentrations of 1 , $10,100 \mathrm{nM}$ with $0.3 \mu \mathrm{M}$ forskolin for $60 \mathrm{~min}$. Values each represent a mean \pm S.E.M. $(\mathbf{n}=5)$. ${ }^{*} \mathbf{P}<0.05$ and ${ }^{* *} \mathrm{P}<0.01$ vs the intracellular dopamine level in the control group. ${ }^{* t /} \mathrm{P}<0.01$ vs the intracellular DOPAC level in the control group. ${ }^{a}$ and ${ }^{b}: P<0.01$ vs the intracellular dopamine and DOPAC levels in the untreated group, respectively.

nM (Fig. 3). The minimum effective concentrations of rolipram on the intracellular dopamine and DOPAC levels were 100 and $10 \mathrm{nM}$, respectively.

We found that a selective PDE4 inhibitor rolipram was effective in promoting dopamine biosynthesis in cultured mesencephalic neurons. Since the selective inhibitors for other PDE tested were not effective, the promoting effect on the forskolin-induced increase of dopamine biosynthesis is likely to be a specific action of the PDE4 inhibitor. In addition, rolipram treatment increased the extracellular level of dopamine. This finding indicates that the effect of rolipram on intracellular dopamine and DOPAC is not the inhibition of dopamine release but promotion of dopamine biosynthesis. This phenomenon may be caused by the increased amount of dopamine in synaptic vesicles following the elevated dopamine biosynthesis. Rolipram at $1 \mu \mathrm{M}$ slightly increased the extracellular level of dopamine under the forskolin-free condition. In this case, rolipram seems to promote dopamine release by increasing the cAMP level in response to spontaneous synaptical transmission. However, the increase of dopamine biosynthesis in the dopaminergic neurons treated with rolipram compared to the control group was far larger under the adenylyl cyclase stimulated condition than under the unstimulated condition. Thus, a characteristic effect of rolipram was that it hardly affected the basal dopamine biosynthesis but enhanced forskolin-induced dopamine biosynthesis. This means that rolipram was effective when the neurons were activated by cAMP- dependent signal transductions.

Rolipram was previously reported to inhibit PDE4 in cortical slices at a micromolar concentration $\left(\mathrm{IC}_{50}=1\right.$ $\mu \mathrm{M})(8)$. Recently, many subtypes of PDE4 were cloned, and the $K_{i}$ or $I_{50}$ of rolipram for the PDE4 subtypes have been reported to be in the range of 0.06 to $2.2 \mu \mathrm{M}$ (9), suggesting the functional heterogeneity in the PDE4 subtypes. On the other hand, rolipram has been found to affect biological functions at a nanomolar concentration: e.g., an enhancement of acid secretion in rabbit gastric glands $\left(\mathrm{EC}_{50}=13 \mathrm{nM}\right)(10)$, inhibition of tumor necrosis factor- $\alpha$ (TNF $\alpha$ ) release from lipopolysaccharide-stimulated macrophages $\left(E C_{50}=12 \mathrm{nM}\right)(11)$, etc. These facts suggest that PDE4 subtypes that show high sensitivity for rolipram play a major role in regulation of intracellular cAMP level in some kinds of cells. In the present study, the minimum effective concentration of rolipram in enhancing dopamine biosynthesis in cultured mesencephalic neurons was $10 \mathrm{nM}$. This finding suggests that PDE4 subtypes showing high sensitivities to rolipram have functional importance in mesencephalic neurons.

Vinpocetine caused marked depletion of dopamine in the dopaminergic neurons. These effects were similar to the effect of W-7 in our finding and to the reported effects of calmodulin antagonists which increase noradrenaline release from permeated synaptosomes (12). Since vinpocetine had been reported to inhibit PDE1 without camodulin-inhibition (13), the depletion of dopamine caused by vinpocetine seems to be by way of direct PDE1 inhibition. These findings suggest that PDE1 regulates the release of dopamine or other neurotransmitters, whereas PDE4 regulates the intracellular cAMP level to control the dopamine biosynthesis in mesencephalic neurons.

In conclusion, we have shown that the PDE4 inhibitor rolipram is effective in promoting forskolin-induced dopamine biosynthesis in mesencephalic neurons treated with forskolin at a concentration of $10 \mathrm{nM}$ or more. The present findings suggest that PDE4 showing high-sensitivity for rolipram plays a major role in the regulation of intracellular cAMP in the dopaminergic neurons.

\section{Acknowledgments \\ We thank Dr. Kazuho Abe (University of Tokyo) for his valuable discussions and Ms. Mika Yamamoto for her technical contribution.}

\section{REFERENCES}

1 Beavo JA: Cyclic nucleotide phosphodiesterases: functional implications of multiple isoforms. Physiol Rev 75, 725-748 (1995)

2 Reinhardt RR and Bondy CA: Differential cellular pattern of gene expression for two distinct cGMP-inhibited cyclic nucleotide phosphodiesterases in developing and mature rat brain. Neuroscience 72, 567-578 (1996)

3 Schoffelmeer AN, Wardeh $G$ and Mulder AH: Cyclic AMP 
facilitates the electrically evoked release of radiolabelled noradrenaline, dopamine and 5-hydroxytryptamine from rat brain slices. Naunyn Schmiedebergs Arch Pharmacol 330, 74-76 (1985)

4 Hulley P, Hartikka J, Abdel'Al S, Engels P, Buerki H-R and Wiederhold $\mathrm{K}-\mathrm{H}$ : Inhibitors of type IV phosphodiesterases reduce the toxicity of MPTP in substantia nigra neurons in vivo. Eur J Neurosci 7, 2431-2440 (1995)

5 Kaufman S: Tyrosine hydroxylase. In Advances in Enzymology and Related Areas of Molecular Biology, Edited by Meister A, Vol 70, pp 103-220, John Wiley \& Sons, New York (1995)

6 Stoclet J-C, Keravis T, Komas N and Lugnier C: Cyclic nucleotide phosphodiesterases as therapeutic targets in cardiovascular diseases. Exp Opin Invest Drugs 4, 1081-1100 (1995)

7 Takeda H: Detection and identification modes for the highly sensitive and simultaneous determination of various biogenic amines by coulometric high-performance liquid chromatography. J Chromatogr 515, 265-278 (1990)

8 Davis CW: Assessment of selective inhibition of rat cerebral cortical calcium-dependent and calcium-independent phosphodiesterases in crude extracts using deoxy-cyclic AMP and potassium ions. Biochem Biophys Acta 797, 354-362 (1984)
9 Müler T, Engels P and Fozard JR: Subtypes of the type 4 CAMP phosphodiesterases: structure, regulation and selective inhibition. Trends Pharmacol Sci 17, 294-298 (1996)

10 Barnette MS, Grous M, Cieslinski LB, Burman M, Christensen SB and Torphy TJ: Inhibitors of phosphodiesterase IV (PDE IV) increase acid secretion in rabbit isolated gastric glands: cor relation between function and interaction with a high-affinity rolipram binding site. J Pharmacol Exp Ther 273, 1396-1402 (1995)

11 Genein CP, Robert T, Davis RL, Nguyen MH, Uccelli A, Faulds D, Li Y, Hedgpeth $J$ and Hauser SL: Prevention of autoimmune demyelination in non-human primates by a cAMP-specific phosphodiesterase inhibitor. Proc Natl Acad Sci USA 92, 3601-3605 (1995)

12 Hens JJ, Oestreicher AB, De Wit M, Marquart A, Gispen WH and De Graan PN: Evidence for a role of calmodulin in calcium-induced noradrenaline release from permeated synaptosomes: effects of calmodulin antibodies and antagonists. J Neurochem 66, 1933-1942 (1996)

13 Hagiwara $M$, Endo $T$ and Hidaka $H$ : Effects of vinpocetine on cyclic nucleotide mechanism in vascular muscle. Biochem Pharmacol 33, $453-457$ (1984) 